\title{
Teaching otherwise: to learn better
}

Saloua Fenniche Trabelsi

Saloua Fenniche Trabelsi, "Teaching otherwise: to learn better," Proc. SPIE 9664, Ninth International Topical Meeting on Education and Training in Optics and Photonics, 966406 (24 October 2005); doi: 10.1117/12.2207787 and Photonics, 2005, Marseille, France 


\title{
Ref ETOP102
}

\section{Teaching otherwise...to learning better}

\author{
Saloua FENNICHE TRABELSI
}

\begin{abstract}
s
It means to do: on the one hand, a comparative study between teaching in the beginning of $X X$ th century and now, on the other hand a comparative study between teaching with a problem and teaching with the predictions (teaching of physical generally and optic particularly).

The first study will be done from a text righted in 1920 witch the author put the accent about what can the school offer to the pupils and what the pupils expect from the school.

1ère partie:

\section{Summary :}

- présentation du texte

- présentation des grandes lignes de la recherche auprés $d l^{\prime}$ un certain nombre dl'enseignants

- présenter les résultats de la recherche

- commentaires

2ème partie

- apercu bref sur II' état actuel de Il'enseignement de ll'optique dans les écoles et les lycées

- remédiations: * I'enseignement par le problème: débuter une séance de cours ou de TP par une situation problème de la vie courante, ll'apprenant sera confroté à un obstacle qul'il ne peut dépasser qul'aprés ( ou au cours de ) la séance.

* Il'enseignement par prédictions: On adopte ici la méthode expérimentale à propement parlé; en effet on débute la séance par un prétest dans lequel Il'apprenant essaye de prédire des réponses à certaines questions et dl'émettre des hypothèses concernant certains faits réels ou expérimentaux, ensuite Il'expérience confirmera ou infirmera ces réponses.
\end{abstract}

\begin{abstract}
s
II sl'agit de faire, dl'une part, une étude comparative de Il'enseignement au début du siècle passé et celui dl'aujourdl'hui et dl'autre part de ll'enseignement par le problème et celui par les prédictions (enseignement de la physique en général et celui de II'optique en particulier). La première étude se fera à partir dl'un texte écrit en 1920 dans lequel II'auteur met II'accent sur ce qul'offre Il'école à Il'enfant et ce à quoi Il'enfant aspire. Cette intervention se basera essentiellement sur les résultats dl'une recherche faite auprés des enseignants des collèges et des lycées à ce propos.
\end{abstract}

